\title{
Singular potential Hamiltonian system
}

Tacksun Jung ${ }^{1}$ and Q-Heung Choi ${ }^{2^{*}}$

"Correspondence:

qheung@inha.ac.kr

2Department of Mathematics

Education, Inha University, Incheon,

402-751, Korea

Full list of author information is

available at the end of the article

\section{Abstract}

We investigate multiple solutions for the Hamiltonian system with singular potential nonlinearity and periodic condition. We get a theorem which shows the existence of the nontrivial weak periodic solution for the Hamiltonian system with singular potential nonlinearity. We obtain this result by using the variational method, critical point theory for indefinite functional.

MSC: 35070

Keywords: Hamiltonian system; singular potential nonlinearity; variational method; critical point theory for indefinite functional; (P.S.) c condition

\section{Introduction}

Let $D$ be an open subset in $R^{2 n}$ with a compact complement $C=R^{2 n} \backslash D, n \geq 2$. Let $p \in R^{n}$, $q \in R^{n},(p(t), q(t)) \in C^{2}([0,2 \pi], D)$ and $G(t,(p(t), q(t))) \in C^{2}\left([0,2 \pi] \times D, R^{1}\right)$. In this paper we investigate the number of solutions $(p(t), q(t)) \in C^{2}([0,2 \pi], D)$ with singular potential nonlinearity and periodic condition

$$
\begin{aligned}
& \dot{p(t)}=-G_{q}(t, p(t), q(t)), \\
& \dot{q(t)}=G_{p}(t, p(t), q(t)), \\
& p(0)=p(2 \pi), \quad q(0)=q(2 \pi) .
\end{aligned}
$$

Let us set $z=(p, q)$ and

$$
J=\left(\begin{array}{cc}
0 & -I_{n} \\
I_{n} & 0
\end{array}\right)
$$

Then (1.1) can be rewritten as

$$
\begin{aligned}
& -J \dot{z}=G_{z}(t, z(t)), \\
& z(0)=z(2 \pi),
\end{aligned}
$$

where $\dot{z}=\frac{d z}{d t}$. We assume that $G(t, z(t)) \in C^{2}\left([0,2 \pi] \times D, R^{1}\right)$ satisfies the following conditions:

(G1) There exists $R_{0}>0$ such that

$$
\sup \left\{|G(t, z(t))|+\left\|\operatorname{grad}_{z} G(t, z(t))\right\|_{R^{2 n}} \mid(t, z) \in[0,2 \pi] \times\left(R^{2 n} \backslash B_{R_{0}}\right)\right\}<+\infty
$$


(G2) There is a neighborhood $Z$ of $C$ in $R^{2 n}$ such that

$$
G(t, z) \geq \frac{A}{d^{2}(z, C)} \quad \text { for }(t, z) \in[0,2 \pi] \times Z
$$

where $d(z, C)$ is the distance function to $C$ and $A>0$ is a constant.

Several authors ([1-4], etc.) studied the Hamiltonian system with nonsingular potential nonlinearity. Jung and Choi $[2,3]$ considered (1.1) with nonsingular potential nonlinearity or jumping nonlinearity crossing one eigenvalue, or two eigenvalues, or several eigenvalues. Chang [1] proved that (1.1) has at least two nontrivial $2 \pi$-periodic weak solutions under some asymptotic nonlinearity. Jung and Choi [2] proved that (1.1) has at least $m$ weak solutions, which are geometrically distinct and nonconstant under some jumping nonlinearity.

In this paper we are trying to find the weak solutions $z \in C^{2}([0,2 \pi], D)$ of system (1.2) such that

$$
\int_{0}^{2 \pi} \dot{z} \cdot w-J G_{z}(t, z(t)) \cdot J w d t=0 \quad \text { for all } w \in E
$$

i.e.,

$$
\int_{0}^{2 \pi}\left[\left(\dot{p}+G_{q}(t, z(t))\right) \cdot \psi-\left(\dot{q}-G_{p}(t, z(t))\right) \cdot \phi\right] d t=0 \quad \text { for all } \zeta=(\phi, \psi) \in E,
$$

where $E$ is introduced in Section 2.

Our main result is as follows.

Theorem 1.1 Assume that $G$ satisfies conditions (G1)-(G2). Then system (1.1) has at least one $2 \pi$-periodic solution.

For the proof of Theorem 1.1, we introduce the perturbed operator $A_{\epsilon}$, such that $A_{\epsilon}^{-1}$ is a compact operator and the associated functional $I(z)$ corresponding to the operator $A_{\epsilon}$, and approach the variational method, the critical point theory. In Section 2, we investigate the Fréchet differentiability of the associated functional $I(z)$ and recall the critical point theorem for indefinite functional. In Section 3, we show that the associated functional $I(z)$ satisfies the geometrical assumptions of the critical point theorem for indefinite functional and prove Theorem 1.1.

\section{Variational method}

Let $L^{2}\left([0,2 \pi], R^{2 n}\right)$ denote the set of $2 n$-tuples of the square integrable $2 \pi$-periodic functions and choose $z \in L^{2}\left([0,2 \pi], R^{2 n}\right)$. Then it has a Fourier expansion $z(t)=\sum_{k=-\infty}^{k=+\infty} a_{k} e^{i k t}$, with $a_{k}=\frac{1}{2 \pi} \int_{0}^{2 \pi} z(t) e^{-i k t} d t \in C^{2 n}, a_{-k}=\bar{a}_{k}$ and $\sum_{k \in Z}\left|a_{k}\right|^{2}<\infty$. Let

$$
A: z(t) \mapsto-J \dot{z}(t)
$$

with the domain

$$
\begin{aligned}
D(A) & =\left\{z(t) \in H^{1}\left([0,2 \pi], R^{2 n}\right) \mid z(0)=z(2 \pi)\right\} \\
& =\left\{\left.z(t) \in L^{2}\left([0,2 \pi], R^{2 n}\right)\left|\sum_{k \in Z}(\epsilon+|k|)^{2}\right| a_{k}\right|^{2}<+\infty\right\},
\end{aligned}
$$


where $\epsilon$ is a positive small number. Then $A$ is a self-adjoint operator. Let $\left\{M_{\lambda}\right\}$ be the spectral resolution of $A$, and let $\alpha$ be a positive number such that $\alpha \notin \sigma(A)$ and $[-\alpha, \alpha]$ contains only one element 0 of $\sigma A$. Let

$$
P_{0}=\int_{-\alpha}^{\alpha} d M_{\lambda}, \quad P_{+}=\int_{\alpha}^{+\infty} d M_{\lambda}, \quad P_{-}=\int_{-\infty}^{-\alpha} d M_{\lambda}
$$

Let

$$
L_{0}=P_{0} L^{2}\left([0,2 \pi], R^{2 n}\right), \quad L_{+}=P_{+} L^{2}\left([0,2 \pi], R^{2 n}\right), \quad L_{-}=P_{-} L^{2}\left([0,2 \pi], R^{2 n}\right) .
$$

For each $u \in L^{2}\left([0,2 \pi], R^{2 n}\right)$, we have the composition

$$
u=u_{0}+u_{+}+u_{-}
$$

where $u_{0} \in L_{0}, u_{+} \in L_{+}, u_{-} \in L_{-}$. According to $A$, there exists a small number $\epsilon>0$ such that $-\epsilon \notin \sigma(A)$. Let us define the space $E$ as follows:

$$
E=D\left(|A|^{\frac{1}{2}}\right)=\left\{\left.z \in L^{2}\left([0,2 \pi], R^{2 n}\right)\left|\sum_{k \in Z}(\epsilon+|k|)\right| a_{k}\right|^{2}<\infty\right\}
$$

with the scalar product

$$
(z, w)_{E}=\epsilon(z, w)_{L^{2}}+\left(|A|^{\frac{1}{2}} z,|A|^{\frac{1}{2}} w\right)_{L^{2}}
$$

and the norm

$$
\|z\|=(z, z)_{E}^{\frac{1}{2}}=\left(\sum_{k \in Z}(\epsilon+|k|)\left|a_{k}\right|^{2}\right)^{\frac{1}{2}} .
$$

The space $E$ endowed with this norm is a real Hilbert space continuously embedded in $L^{2}\left([0,2 \pi], R^{2 n}\right)$. The scalar product in $L^{2}$ naturally extends as the duality pairing between $E$ and $E^{\prime}=W^{-\frac{1}{2}, 2}\left([0,2 \pi], R^{2 n}\right)$. We note that the operator $(\epsilon+|A|)^{-1}$ is a compact linear operator from $L^{2}\left([0,2 \pi], R^{2 n}\right)$ to $E$ such that

$$
\left((\epsilon+|A|)^{-1} w, z\right)_{E}=\int_{0}^{2 \pi}(w(t), z(t)) d t
$$

Let

$$
A_{\epsilon}=\epsilon I+A .
$$

Let

$$
E_{0}=\left|A_{\epsilon}\right|^{-\frac{1}{2}} L_{0}, \quad E_{+}=\left|A_{\epsilon}\right|^{-\frac{1}{2}} L_{+}, \quad E_{-}=\left|A_{\epsilon}\right|^{-\frac{1}{2}} L_{-} .
$$

Then $E=E_{0} \oplus E_{+} \oplus E_{-}$and for $z \in E$, $z$ has the decomposition $z=z_{0}+z_{+}+z_{-} \in E$, where

$$
z_{0}=\left|A_{\epsilon}\right|^{-\frac{1}{2}} u_{0}, \quad z_{+}=\left|A_{\epsilon}\right|^{-\frac{1}{2}} u_{+}, \quad z_{-}=\left|A_{\epsilon}\right|^{-\frac{1}{2}} u_{-} .
$$


Thus we have

$$
\left\|z_{0}\right\|_{E_{0}}=\left\|u_{0}\right\|_{L_{0}}, \quad\left\|z_{+}\right\|_{E_{+}}=\left\|u_{+}\right\|_{L_{+}}, \quad\left\|z_{-}\right\|_{E_{-}}=\left\|u_{-}\right\|_{L_{-}}
$$

and that $E_{0}, E_{+}, E_{-}$are isomorphic to $L_{0}, L_{+}, L_{-}$, respectively.

For the sake of simplicity, from now on we shall denote the subset of $C^{2}([0,2 \pi], D)$, satisfying the $2 \pi$-periodic condition, by $C^{2}\left(S^{1}, D\right)$.

Let us introduce an open set of the Hilbert space $E$ as follows:

$$
X=\left\{z \in E \mid z(t) \in D \subset R^{2 n}, t \in S^{1}\right\}
$$

Let

$$
X_{0}=E_{0} \cap X, \quad X_{+}=E_{+} \cap X, \quad X_{-}=E_{-} \cap X .
$$

Then $X=X_{0} \oplus X_{+} \oplus X_{-}$and for $z \in X, z$ has the decomposition $z=z_{0}+z_{+}+z_{-} \in X$, where

$$
z_{0} \in X_{0}, \quad z_{+} \in X_{+}, \quad z_{-} \in X_{-} .
$$

The associated functional of (1.2) on $X$ is as follows:

$$
I(z)=\frac{1}{2}\left(\left\|A_{\epsilon}^{\frac{1}{2}} z_{+}\right\|_{L^{2}}^{2}+\left\|A_{\epsilon}^{\frac{1}{2}} M_{+} z_{0}\right\|^{2}-\left\|\left(-A_{\epsilon}\right)^{\frac{1}{2}} z_{-}\right\|_{L^{2}}^{2}-\left\|\left(-A_{\epsilon}\right)^{\frac{1}{2}} M_{-} z_{0}\right\|^{2}\right)-\psi_{\epsilon}(z),
$$

where $\psi_{\epsilon}(z)=\psi(z)+\frac{\epsilon}{2}\|z\|_{L^{2}}^{2}, \psi(z)=\int_{0}^{2 \pi} G(t, z(t)) d t$. Let

$$
F(z)=G_{z}(t, z(t))
$$

By $G \in C^{2}, \psi(z)=\int_{0}^{2 \pi} G(t, z(t)) \in C^{2}\left(S^{1} \times D, R^{1}\right)$. Let

$$
F_{\epsilon}(z)=\epsilon I+F(z)=\epsilon I+G_{z}(t, z(t)) \text {. }
$$

System (1.2) can be rewritten as

$$
A_{\epsilon}(z)=F_{\epsilon}(z) \text {. }
$$

The Euler equation of the functional $I(z)$ is the system

$$
\begin{aligned}
& u_{+}=\left|A_{\epsilon}\right|^{-\frac{1}{2}} P_{+} F_{\epsilon}(z), \\
& u_{-}=-\left|A_{\epsilon}\right|^{-\frac{1}{2}} P_{-} F_{\epsilon}(z), \\
& M_{+} u_{0}=\left|A_{\epsilon}\right|^{-\frac{1}{2}} M_{+} P_{0} F_{\epsilon}(z), \quad M_{-} u_{0}=-\left|A_{\epsilon}\right|^{-\frac{1}{2}} M_{-} P_{0} F_{\epsilon}(z) .
\end{aligned}
$$

Thus $z=z_{0}+z_{+}+z_{-}$is a solution of (2.2) if and only if $u=u_{0}+u_{+}+u_{-}$is a critical point of $I$. System (2.3)-(2.5) is reduced to

$$
A_{\epsilon} z_{+}=P_{+} F_{\epsilon}\left(z_{0}+z_{+}+z_{-}\right) \quad \text { or } \quad z_{+}=\left(A_{\epsilon}\right)^{-1} P_{+} F_{\epsilon}\left(z_{0}+z_{+}+z_{-}\right)
$$




$$
\begin{aligned}
& A_{\epsilon} z_{-}=P_{-} F_{\epsilon}\left(z_{0}+z_{+}+z_{-}\right) \quad \text { or } \quad z_{-}=\left(A_{\epsilon}\right)^{-1} P_{-} F_{\epsilon}\left(z_{0}+z_{+}+z_{-}\right) \\
& A_{\epsilon} M_{+} z_{0}=M_{+} P_{0} F_{\epsilon}\left(z_{0}+z_{+}+z_{-}\right), \quad A_{\epsilon} M_{-} z_{0}=M_{-} P_{0} F_{\epsilon}\left(z_{0}+z_{+}+z_{-}\right) .
\end{aligned}
$$

By the condition $F(z) \in C^{2}(E, E)$ and $(\mathrm{G} 2)$,

$$
\left\|F_{\epsilon}(z)-F_{\epsilon}(w)\right\|_{L^{2}} \leq(\epsilon+\beta)\|u-v\|_{H} \quad \forall u, v \in E
$$

By (G3) and (G4), there exists $\gamma>\min \left\{j_{1}-\alpha+\epsilon, \beta-j_{1}+\epsilon\right\}$ such that

$$
\left\|\left.A_{\epsilon}^{-1}\right|_{L_{+} \oplus L_{-}}\right\| \leq \frac{1}{\gamma}
$$

By the following lemma, the weak solutions of (1.2) coincide with the critical points of the functional $I(z)$.

Lemma 2.1 Assume that G satisfies conditions (G1)-(G2). Then $I(z)$ is continuous and Fréchet differentiable in $X$ with the Fréchet derivative

$$
D I(z) w=\int_{0}^{2 \pi}\left(A_{\epsilon} z-F_{\epsilon}(z)\right) \cdot w d t \quad \text { for all } w \in X
$$

Moreover, $D I \in C$. That is, $I \in C^{1}$.

Proof First we prove that $I(z)=\int_{0}^{2 \pi}\left[\frac{1}{2} A_{\epsilon} z-G(t, z(t))-\frac{\epsilon}{2} z^{2}\right] d t$ is continuous and Fréchet differentiable in $X$. For $z, w \in X$,

$$
\begin{aligned}
& |I(z+w)-I(z)| \\
& =\mid \int_{0}^{2 \pi} \frac{1}{2} A_{\epsilon}(z+w) \cdot(z+w)-\int_{0}^{2 \pi}\left[G(t, z+w)+\frac{\epsilon}{2}(z+w)^{2}\right] \\
& \quad-\int_{0}^{2 \pi} \frac{1}{2} A_{\epsilon}(z) \cdot z+\int_{0}^{2 \pi}\left[G(t, z)+\frac{\epsilon}{2} z^{2}\right] \mid \\
& =\mid \int_{0}^{2 \pi} \frac{1}{2}\left[A_{\epsilon}(z) \cdot w+A_{\epsilon}(w) \cdot z+A_{\epsilon}(w) \cdot w\right] \\
& \quad-\int_{0}^{2 \pi}\left[G(t, z+w)-G(t, z)+\frac{\epsilon}{2}\left(2 z \cdot w+w^{2}\right)\right] \mid .
\end{aligned}
$$

We have

$$
\begin{aligned}
& \left|\int_{0}^{2 \pi}[G(t, z+w)-G(t, z)] d t\right| \\
& \quad \leq\left|\int_{0}^{2 \pi}\left[G_{z}(t, z(t)) \cdot w+O\left(\|w\|_{R^{2 n}}\right)\right] d t\right|=O\left(\|w\|_{R^{2 n}}\right) .
\end{aligned}
$$

Thus we have

$$
|I(z+w)-I(z)|=O\left(\|w\|_{R^{2 n}}\right) .
$$


Next we shall prove that $I(z)$ is Fréchet differentiable in $X$. For $z, w \in X$,

$$
\begin{aligned}
|I(z+w)-I(z)-D I(z) w| \\
=\mid \int_{0}^{2 \pi} \frac{1}{2} A_{\epsilon}(z+w) \cdot(z+w)-\int_{0}^{2 \pi}\left[G(t, z+w)+\frac{\epsilon}{2}(z+w)^{2}\right] \\
\quad-\int_{0}^{2 \pi} \frac{1}{2} A_{\epsilon}(z) \cdot z+\int_{0}^{2 \pi}\left[G(t, z)+\frac{\epsilon}{2} z^{2}\right] \\
\quad-\int_{0}^{2 \pi} A_{\epsilon}(z) \cdot w+\int_{0}^{2 \pi}\left[G_{z}(t, z)+\epsilon z \cdot w\right] \mid \\
=\mid \int_{0}^{2 \pi} \frac{1}{2}\left[A_{\epsilon}(w) \cdot z+A_{\epsilon}(w) \cdot w\right]-\int_{0}^{2 \pi}\left[G(t, z+w)-G(t, z)-G_{z}(t, z)+\frac{\epsilon}{2} w^{2}\right] .
\end{aligned}
$$

By (2.10), we have

$$
\int_{0}^{2 \pi}\left[G(t, z+w)-G(t, z)-G_{z}(t, z)\right] d t=O\left(\|w\|_{R^{2 n}}\right) .
$$

Thus

$$
|I(z+w)-I(z)-D I(z) w|=O\left(\|w\|_{R^{2 n}}\right) .
$$

Lemma 2.2 Assume that G satisfies conditions (G1)-(G2). Let $\left\{z_{k}\right\} \subset X_{-}$and $z_{k} \rightarrow z$ weakly in $X$ with $z \in \partial X$. Then $I\left(z_{k}\right) \rightarrow-\infty$.

Proof To prove the conclusion, it suffices to prove that

$$
\int_{0}^{2 \pi} G\left(t, z_{k}(t)\right) d t \longrightarrow+\infty
$$

Since $G(t, z(t))$ is bounded from below, it suffices to prove that there is an interval $[a, b] \subset$ $[0,2 \pi]$ such that

$$
\int_{a}^{b} G\left(t, z_{k}(t)\right) d t \longrightarrow+\infty
$$

By definition, $z \in \partial X$ means that there exists $t^{*} \in[0,2 \pi]$ such that $z\left(t^{*}\right) \in \partial D$. By $\mathrm{G}(2)$, there exists a constant $B>0$ such that

$$
G(x, t, U) \geq \frac{A}{d^{2}(z, C)}-B .
$$

Thus we have

$$
\int_{t^{*}}^{t^{*}+\delta} G(t, z(t)) d t \geq \int_{t^{*}}^{t^{*}+\delta}\left(\frac{A}{\left\|z(t)-z\left(t^{*}\right)\right\|_{R^{2 n}}^{2}}-B\right) d t
$$

for all $\delta>0$. By Schwarz's inequality, we have

$$
\left\|z(t)-z_{k}(t)\right\|_{R^{2 n}} \leq\left|t-t^{*}\right|^{\frac{1}{2}}\left(\int_{0}^{2 \pi}\|\dot{z}(t)\|_{R^{2 n}}^{2} d t\right)^{\frac{1}{2}} .
$$


Thus we have

$$
\int_{t^{*}}^{t^{*}+\delta} G(t, z(t)) d t=+\infty .
$$

Since the embedding $H^{1}\left(S^{1}, R^{2 n}\right) \hookrightarrow C\left(S^{1}, R^{2 n}\right)$ is compact, we have

$$
\max \left\{\left\|z(t)-z_{k}(t)\right\|_{R^{2 n}}^{2} \mid t \in S^{1}\right\} \longrightarrow 0 \quad \text { as } k \rightarrow \infty
$$

Thus by Fatou's lemma, we have

$$
\begin{aligned}
\liminf \int_{t^{*}}^{t^{*}+\delta} G\left(t, z_{k}(t)\right) d t & \geq \int_{t^{*}}^{t^{*}+\delta} \liminf G\left(t, z_{k}(t)\right) \\
& =\int_{t^{*}}^{t^{*}+\delta} G(t, z(t)) d t=+\infty
\end{aligned}
$$

Thus

$$
\liminf \int_{t^{*}}^{t^{\prime \prime}+\delta} G\left(t, z_{k}(t)\right)=+\infty
$$

Thus if $\left\{z_{k}\right\} \subset X_{-}$,

$$
\begin{aligned}
I\left(z_{k}\right)= & \int_{0}^{2 \pi}\left[\frac{1}{2} A_{\epsilon} z_{k}(t)-\psi_{\epsilon}\left(z_{k}\right)\right] d x d t \\
= & \frac{1}{2}\left(\left\|A_{\epsilon}^{\frac{1}{2}}\left(z_{k}\right)_{+}\right\|_{L^{2}}^{2}+\left\|A_{\epsilon}^{\frac{1}{2}} M_{+}\left(z_{k}\right)_{0}\right\|^{2}-\left\|\left(-A_{\epsilon}\right)^{\frac{1}{2}}\left(z_{k}\right)_{-}\right\|_{L^{2}}^{2}\right. \\
& \left.-\left\|\left(-A_{\epsilon}\right)^{\frac{1}{2}} M_{-}\left(z_{k}\right)_{0}\right\|^{2}\right)-\psi_{\epsilon}\left(z_{k}\right) \\
\leq & -\left\|\left(-A_{\epsilon}\right)^{\frac{1}{2}}\left(z_{k}\right)_{-}\right\|_{L^{2}}^{2}-\psi_{\epsilon}\left(z_{k}\right) \longrightarrow-\infty,
\end{aligned}
$$

so we prove the lemma.

Now, we recall the critical point theorem for indefinite functional (cf. [5]).

Let

$$
\begin{aligned}
& B_{r}=\{u \in X \mid\|u\| \leq r\}, \\
& S_{r}=\{u \in X \mid\|u\|=r\} .
\end{aligned}
$$

Theorem 2.1 (Critical point theorem for indefinite functional) Let $X$ be a real Hilbert space with $X=X_{1} \oplus X_{2}$ and $X_{2}=X_{1}^{\perp}$. Suppose that $I \in C^{1}(X, R)$ satisfies $(P S)$ and

(I1) $I(u)=\frac{1}{2}(L u, u)+b u$, where $L u=L_{1} P_{1} u+L_{2} P_{2} u$ and $L_{i}: X_{i} \rightarrow X_{i}$ is bounded and self-adjoint, $i=1,2$,

(I2) $b^{\prime}$ is compact, and

(I3) there exists a subspace $\tilde{X} \subset X$ and sets $S \subset X, Q \subset \tilde{X}$ and constants $\alpha>\omega$ such that

(i) $S \subset X_{1}$ and $\left.I\right|_{S} \geq \alpha$,

(ii) $Q$ is bounded and $\left.I\right|_{\partial Q} \leq \omega$,

(iii) $S$ and $\partial Q$ link.

Then I possesses a critical value $c \geq \alpha$. 


\section{Proof of Theorem 1.1}

We shall show that the functional $I(z)$ satisfies the geometric assumptions of the critical point theorem for indefinite functional.

Lemma 3.1 (Palais-Smale condition) Assume that G satisfies conditions (G1) and (G2). Then there exists a constant $\gamma_{0}$ depending on the $C^{1}$ norm of the function $G$ on $S^{1} \times$ $\left(R^{2 n} \backslash B_{R_{0}}\right)$ such that $I(z)$ satisfies the (P.S.) $)_{\gamma}$ condition in $X$ for $\gamma<\gamma_{0}$.

Proof We shall prove the lemma by contradiction. We suppose that there exists a sequence $\left\{z_{k}\right\} \subset X$ satisfying

$$
I\left(z_{k}\right) \rightarrow \gamma
$$

and

$$
D I\left(z_{k}\right)=z_{k}-A_{\epsilon}^{-1}\left(G_{z}\left(t, z_{k}\right)+\epsilon z_{k}\right) \longrightarrow \theta,
$$

where $A_{\epsilon}^{-1}$ is a compact operator and $\theta=(0, \ldots, 0)$. Since $G(t, z(t))$ is bounded from below, (3.1) implies that there exists a constant $C_{1}>0$ such that

$$
\int_{0}^{2 \pi} A_{\epsilon} z_{k}(t) d t \geq C_{1}
$$

We claim that $\left\{z_{k}\right\}$ has a convergent subsequence. It suffices to prove that the sequence $\left\{z_{k}\right\}$ is bounded in $X$. By contradiction, we suppose that up to a subsequence, $\left\{z_{k}\right\}$ satisfies $\left\|z_{k}\right\|_{R^{2 n}} \rightarrow \infty$. Then, for large $k$, we have

$$
\left\|z_{k}\right\|_{R^{2 n}} \geq R_{0}
$$

It follows from (3.4) that

$$
\left|\int_{0}^{2 \pi} G\left(t, z_{k}\right) d t\right| \leq 2 \pi \sup \left\{\left|G\left(t, z_{k}\right)\right| \mid\left(t, z_{k}\right) \in S^{1} \times\left(R^{2 n} \backslash B_{R_{0}}\right)\right\} .
$$

By (3.3) and (3.5),

$$
\begin{aligned}
I\left(z_{k}\right) & =\int_{0}^{2 \pi}\left[\frac{1}{2} A_{\epsilon}\left(z_{k}\right)-G\left(t, z_{k}(t)\right)-\frac{\epsilon}{2} z_{k}^{2}\right] d t \\
& \geq \frac{C_{1}}{2}-2 \pi \sup \left\{\left|G\left(t, z_{k}\right)\right| \mid\left(t, z_{k}\right) \in S^{1} \times\left(R^{2 n} \backslash B_{R_{0}}\right)\right\}-\frac{\epsilon}{2}\left\|z_{k}\right\|_{L^{2}}^{2} .
\end{aligned}
$$

Letting

$$
\gamma_{0}=\frac{C_{1}}{2}-2 \pi \sup \left\{\left|G\left(t, z_{k}\right)\right| \mid\left(t, z_{k}\right) \in S^{1} \times\left(R^{2 n} \backslash B_{R_{0}}\right)\right\}-\frac{\epsilon}{2}\left\|z_{k}\right\|_{L^{2}}^{2},
$$

we have $I\left(z_{k}\right) \geq \gamma_{0}$, which is a contradiction.

Let

$$
Q=\left(\bar{B}_{r} \cap X^{-}\right) \oplus\left\{r e \mid e \in B_{1} \cap X^{+}, 0<r<R\right\} .
$$


Lemma 3.2 Assume that $G$ satisfies conditions (G1) and (G2). Then there exist sets $S_{\rho} \subset$ $X^{+}$with radius $\rho>0, Q \subset X$ and a constant $\alpha>0$ such that

(i) $S_{\rho} \subset X^{+}$and $\left.I\right|_{S_{\rho}} \geq \alpha$,

(ii) $Q$ is bounded and $\left.I\right|_{\partial Q} \leq 0$,

(iii) $S_{\rho}$ and $\partial Q$ link.

Proof (i) Let us choose $z \in X^{+} \subset X$. Then $z(t) \in D$. By (G1), $G(t, z)$ is bounded above and there exists a constant $\tau>0$

$$
\begin{aligned}
I(z)= & \frac{1}{2}\left(\left\|A_{\epsilon}^{\frac{1}{2}} z_{+}\right\|_{L^{2}}^{2}+\left\|A_{\epsilon}^{\frac{1}{2}} M_{+} z_{0}\right\|-\left\|\left(-A_{\epsilon}\right)^{\frac{1}{2}} z_{-}\right\|_{L^{2}}^{2}-\left\|\left(-A_{\epsilon}\right)^{\frac{1}{2}} M_{-} z_{0}\right\|^{2}\right) \\
& -\int_{0}^{2 \pi} G(t, z) d t-\frac{\epsilon}{2}\|z\|_{L^{2}}^{2} \\
\geq & \frac{1}{2}\left\|A_{\epsilon}^{\frac{1}{2}} z_{+}\right\|_{L^{2}}^{2}-\tau-\frac{\epsilon}{2}\|z\|_{L^{2}}^{2}
\end{aligned}
$$

for $\tau>0$. Then there exist constants $\rho>0$ and $\alpha>0$ such that if $z \in S_{\rho} \cap X^{+}$, then $I(z) \geq \alpha$.

(ii) Let us choose $e \in B_{1} \cap X^{+}$. Let $z \in \bar{B}_{r} \cap X^{-} \oplus\{r e \mid 0<r\}$. Then $z=w+y, w \in \bar{B}_{r} \cap X^{-}$, $y=r e$. We note that

$$
\text { If } w \in \bar{B}_{r} \cap X^{-} \text {, then } \int_{0}^{2 \pi} A_{\epsilon}(z) \cdot z d t=-\left\|\left(-A_{\epsilon}\right)^{\frac{1}{2}} z_{-}\right\|_{L^{2}}^{2} \leq 0 \text {. }
$$

By (G2), $G(t, w+r e)$ is bounded from below. Thus, by Lemma 2.2, there exists a constant $\tau>0$ such that if $z=w+r e$, then we have

$$
\begin{aligned}
I(z) & =\frac{1}{2} r^{2}-\left\|\left(-A_{\epsilon}\right)^{\frac{1}{2}} z_{-}\right\|_{L^{2}}^{2}-\int_{0}^{2 \pi} G(t, w+r e) d t-\frac{\epsilon}{2}\|z\|_{L^{2}}^{2} \\
& \leq \frac{1}{2} r^{2}-\left\|\left(-A_{\epsilon}\right)^{\frac{1}{2}} z_{-}\right\|_{L^{2}}^{2}-\int_{\Omega} \frac{\tau}{d^{2}(V+r e, C)} d x d t-\frac{\epsilon}{2}\|z\|_{L^{2}}^{2} .
\end{aligned}
$$

We can choose a constant $R>r$ such that if $z=w+r e \in Q=\left(\bar{B}_{r} \cap X^{-}\right) \oplus\left\{r e \mid e \in B_{1} \cap X^{+}, 0<\right.$ $r<R\}$, then $I(z)<0$. Thus we prove the lemma.

Proof of Theorem 1.1 By Lemma 2.1, $I(z)$ is continuous and Fréchet differentiable in $X$ and, moreover, $D I \in C$. By Lemma 2.2, if $\left\{z_{k}\right\} \subset X^{-}$and $z_{k} \rightarrow z$ weakly in $X$ with $z \in \partial X$, then $I\left(z_{k}\right) \rightarrow-\infty$. By Lemma 3.1, $I(z)$ satisfies the (P.S. $)_{\gamma}$ condition for $\gamma<\gamma_{0}$. By Lemma 3.2, there exist sets $S_{\rho} \subset X^{+}$with radius $\rho>0, Q \subset X$ and a constant $\alpha>0$ such that $\left.I\right|_{S_{\rho}} \geq \alpha, Q$ is bounded and $\left.I\right|_{\partial Q} \leq 0$, and $S_{\rho}$ and $\partial Q$ link. By the critical point theorem, $I(z)$ possesses a critical value $c \geq \alpha$. Thus (1.1) has at least one nontrivial weak solution. Thus we prove Theorem 1.1.

Competing interests

The authors did not provide this information.

Authors' contributions

The authors did not provide this information.

\section{Author details}

${ }^{1}$ Department of Mathematics, Kunsan National University, Kunsan, 573-701, Korea. ${ }^{2}$ Department of Mathematics Education, Inha University, Incheon, 402-751, Korea. 


\section{Acknowledgements}

This work (Tacksun Jung) was supported by Basic Science Research Program through the National Research Foundation of Korea(NRF) funded by the Ministry of Education, Science and Technology (KRF-2010-0023985).

Received: 24 April 2013 Accepted: 8 October 2013 Published: 19 Nov 2013

\section{References}

1. Chang, KC: Infinite Dimensional Morse Theory and Multiple Solution Problems. Birkhäuser, Boston (1993)

2. Jung, T, Choi, $\mathrm{QH}$ : On the number of the periodic solutions of the nonlinear Hamiltonian system. Nonlinear Anal. TMA 71, e1100-e1108 (2009)

3. Jung, T, Choi, QH: Periodic solutions for the nonlinear Hamiltonian systems. Korean J. Math. 17, $331-340$ (2009)

4. Rabinowitz, PH: Minimax Methods in Critical Point Theory with Applications to Differential Equations. CBMS. Regional Conf. Ser. Math., vol. 65. Am. Math. Soc., Providence (1986)

5. Benci, V, Rabinowitz, PH: Critical point theorems for indefinite functionals. Invent. Math. 52, 241-273 (1979)

10.1186/1029-242X-2013-545

Cite this article as: Jung and Choi: Singular potential Hamiltonian system. Journal of Inequalities and Applications 2013, 2013:545

\section{Submit your manuscript to a SpringerOpen ${ }^{\circ}$ journal and benefit from:}

- Convenient online submission

- Rigorous peer review

- Immediate publication on acceptance

Open access: articles freely available online

- High visibility within the field

- Retaining the copyright to your article 